\title{
Six observational pieces of evidence against corotation as the main cause for the aurora at Jupiter
}

\author{
Bertrand Bonfond $^{1}$, Zhonghua $\mathrm{Yao}^{2}$, and Denis Grodent ${ }^{1}$ \\ ${ }^{1}$ LPAP, STAR Institute, Université de Liège, Liège, Belgium (b.bonfond@uliege.be) \\ ${ }^{2}$ Key Laboratory of Earth and Planetary Physics, Institute of Geology and Geophysics, Chinese Academy of Sciences, Beijing, \\ China
}

The Main Emissions are the most recognizable feature of the aurorae at Jupiter and they are responsible for roughly $1 / 3$ rd of the total emitted power. They form an ever-present and quasicontinuous ring of emission centered on the magnetic poles. The most widely accepted explanation for these auroral emissions involves a current system related to the corotation enforcement of the plasma in the Jovian magnetosphere. Models based on this theory explain many characteristics of the aurorae. However, recent observations from the NASA Juno spacecraft and the ESA/NASA Hubble Space Telescope, complemented by previous results from the NASA Galileo spacecraft, challenge this theoretical framework. In this presentation, we will review six specific sets of observations contradictory with expectations from the corotation enforcement theory:

We will expose their implications for the modelling of the Jovian magnetosphere and aurorae and we will discuss promising paths forward. 